\section{IN BRIEF}

\section{$\Rightarrow$ IMMUNOTHERAPY}

\section{TIL infusions effective in HPV-associated cancers}

Adoptive immunotherapy with infusion of ex vivo expanded tumour-infiltrating lymphocytes (TILs) is an attractive approach to anticancer therapy owing to the high levels of antitumour specificity that can be achieved. Now, the outcomes of a phase II study in which patients with human papillomavirus (HPV)-associated cervical $(n=18)$ and noncervical $(n=11)$ cancers reveal that this approach is feasible and is effective in some patients. TIL cultures were expanded ex vivo in the presence of tumour specimens and administered to patients as a single infusion following a nonmyeloablative chemotherapy conditioning regimen. Objective responses were observed in $28 \%$ of patients (5/18) with cervical cancer and in $18 \%(2 / 11)$ without. Two responses were complete and were ongoing at the time of analysis (at 67 and 53 months after infusion). The adverse event profile was consistent with that of the chemotherapy regimen: no infusion-related or autoimmune toxicities were reported.

ORIGINAL ARTICLE Stevanovic, S. et al. A phase Il study of tumor-infiltrating lymphocyte therapy for human papillomavirus-associated epithelial cancers. Clin. Cancer Res. https://doi.org/10.1158/1078-0432.CCR-18-2722 (2018)

\section{$\Rightarrow$ CNS CANCER}

\section{Relapsed glioblastomas respond to regorafenib}

Data from a phase II trial involving patients with relapsed glioblastoma following chemoradiotherapy, a setting in which few evidence-based therapies are currently available, demonstrate the superiority of the anti-angiogenic tyrosinekinase inhibitor regorafenib over lomustine chemotherapy. Patients receiving regorafenib had a median overall survival duration of 7.6 months versus 5.6 months in the lomustine group (HR 0.50, 95\% Cl 0.33-0.75; log-rank $P=0.0009$ ). Grade 3-4 adverse events were more common in the regorafenib group (occurring in $56 \%$ versus $40 \%$ of patients) and the majority were haematological: decreased platelet count, decreased lymphocyte count and neutropenia were observed in $13 \%$, $13 \%$ and $12 \%$ of patients, respectively. No treatment-related deaths were reported in either arm. These findings merit further investigation in a randomized phase III trial.

ORIGINAL ARTICLE Lombardi, G. et al. Regorafenib compared with lomustine in patients with relapsed glioblastoma (REGOMA): a multicentre, open-label, randomised, controlled, phase 2 trial. Lancet https://doi.org/10.1016/S1470-2045(18)30675-2 (2018)

\section{$\Rightarrow$ DRUG THERAPY}

\section{Apixaban reduces risk of thromboembolism}

The results of a randomized controlled trial demonstrate that the novel oral anticoagulant apixaban, administered up to 5 days before the start of chemotherapy, significantly reduces the risk of venous thromboembolism (VTE) in patients with intermediate-risk or high-risk cancers of various types, including lymphomas, gynaecological and pancreatic cancers. Patients were randomized 1:1 to receive either apixaban or placebo, followed by chemotherapy appropriate to their cancer type. A total of 12 patients (4.2\%) in the apixaban group had VTEs, compared with 28 patients (10.2\%) in the placebo group (HR 0.41; 95\% Cl 0.26- 0.65; $P<0.001$ ). This reduction in VTE risk comes at the cost of an increased risk of major bleeding episodes: major bleeding occurred in 10 patients (3.5\%) in the apixaban group versus 5 patients (1.8\%) in the placebo group (HR 2.00; 95\% Cl 1.01-3.95; $P=0.046$ ). However, results of the competing-risk analysis favour the use of apixaban in this patient population (HR 0.42; 95\% $\mathrm{Cl} 0.27-0.65$ ).

ORIGINAL ARTICLE Carrier, M. et al. Apixaban to prevent venous thromboembolism in patients with cancer. N. Engl.J. Med. https://doi.org/10.1056/NEJMoa1814468 (2018)

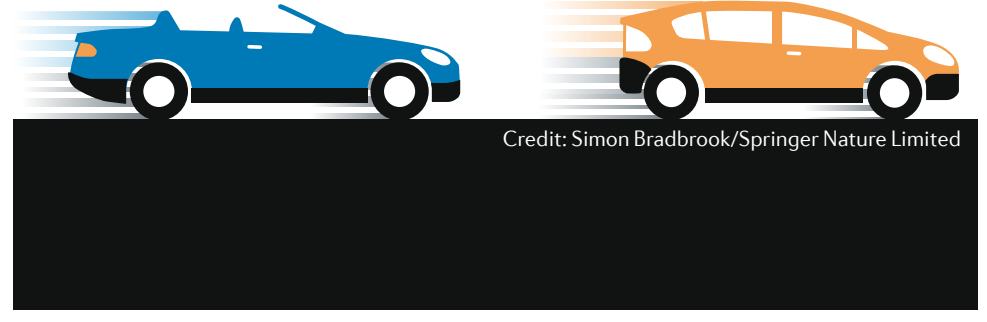

GASTROINTESTINAL CANCER

\title{
Hybrid minimally invasive surgery overtakes open surgery
}

Curative surgery increases the 5 -year survival of patients with oesophageal cancer from $10-15 \%$ overall to $40 \%$, but postoperative morbidities affect more than half of all patients who undergo open oesophagectomy. Minimally invasive oesophagectomy (MIE) approaches have been developed to reduce postoperative pulmonary morbidity. Now, the efficacy of hybrid MIE surgery and open surgery have been compared in the MIRO phase III trial with promising results.

Hybrid MIE combines laparoscopic abdominal gastric mobilization with open thoracotomy. In 2012, the results of the TIME trial showed that total MIE (laparoscopic abdominal phase and thoracoscopic phase) was associated with a lower incidence of complications than open surgery. "However, total MIE is technically demanding and some studies suggest a higher risk of intrathoracic anastomotic leakage," explains lead investigator Guillaume Piessen. Whether the maximal benefit of a MIE approach is mostly associated with the abdominal or thoracic phase needs to be determined in order to optimize the outcomes of oesophagectomy.

In the MIRO trial, patients with resectable squamous cell carcinoma or adenocarcinoma of the middle or lower third of the oesophagus were randomly allocated to undergo hybrid MIE $(n=103)$ or open oesophagectomy $(n=104)$. The incidence of major intraoperative and/or postoperative complications at 30 days was significantly lower with hybrid MIE than with open surgery (36\% versus $64 \%$; $P<0.001$ ).
The incidence of major pulmonary complications within 30 days of surgery was also lower in the hybrid MIE group (18\% versus 30\%). No differences between groups were observed in postoperative mortality at 30 days ( $1 \%$ versus $2 \%)$ or at 90 days (4\% versus $6 \%$ ).

At a median follow-up duration of 48.8 months, median overall survival durations were 52.2 months and 47.6 months with hybrid MIE and open surgery, respectively, although this difference was not statistically significant. Disease-free survival was similar between both groups: $57 \%$ and $48 \%$, respectively, at 3 years and $53 \%$ versus $43 \%$ at 5 years. The pathological analysis revealed similar patterns of tumour histology, stage and nodal involvement between both groups.

"In our study, hybrid MIE was associated with a similar magnitude of benefit in terms of morbidity as that observed with total MIE in other studies, suggesting that the laparoscopic phase is the most critical to decrease morbidity compared with open surgery," comments Piessen. "Our results will not change practice for the small proportion of expert surgeons performing total MIE (20-40\% in Europe and North America), but will surely convince the vast majority of surgeons who still perform open oesophagectomy ( $52 \%$ or probably more)," he concludes.

Diana Romero

ORIGINAL ARTICLE Mariette, C. et al. Hybrid minimally invasive esophagectomy for esophageal cancer. N. Engl.J. Med. 380, 152-162 (2019) FURTHER READING Biere, S. S. A. Y. et al. Minimally invasive versus open oesophagectomy for patients with oesophageal cancer: a multicentre, open-label, randomised controlled trial. Lancet 379, 1887-1892 (2012) 